\title{
Antisense oligonucleotides and magnetic nanoparticles for targeted diagnostic and cancer treatment
}

\begin{abstract}
The resistance of tumors to therapeutic intervention is a major problem in the efficacy of treatments. A recent strategy to overcome this problem proposes the association of antitumor drugs with colloidal nanoparticles. Here a hypothesis is suggested concerning the potential use of antisense Oligo Deoxy Nucleotide (ODN) labeled with iron nanoparticles for tumor antisense gene detection with magnetic resonance gene imaging and cancer treatment.
\end{abstract}

Keywords: antisense therapy, magnetic field, drug delivery, nanoplatform, nanoparticles, nanobiotechnology
Volume I Issue 2 - 2017

\author{
Beatriz López-Díaz, Silvia Mercado-Sáenz, \\ Francisco Sendra-Portero, Miguel J Ruiz- \\ Gómez \\ Department of Radiology and Medicine, University of Málaga, \\ Spain
}

Correspondence: Miguel J Ruiz-Gómez, Department of Radiology and Physical Medicine, University of Málaga, Bulevar Louis Pasteur, 32, 2907I Málaga, Spain, Tel +349 5213 I578, Fax +34952131630,Emailmjrg@uma.es

Received: May 26, 2017 | Published: May 31, 2017

\section{Introduction}

The resistance of tumors to therapeutic intervention is a major problem in the efficacy of treatments. A recent strategy to overcome this problem proposes the association of antitumor drugs with colloidal nanoparticles, with the aim to avoid non-cellular and cellular based mechanisms of resistance and to increase selectivity of drugs. In this way as an example, Wang et al. ${ }^{2}$ reported that targeted magnetic nanoparticles containing 5-FU can improve the chemotherapeutic effect of 5-FU against hepatocellular carcinoma. Nanoparticles may be defined as colloidal systems $(<1 \mu \mathrm{m})$, generally made of polymers. They may act as a drug vehicle able to target tumor cells ${ }^{1}$. The size of the colloidal carriers as well as their surface characteristics are the key for the biological fate of nanoparticles. The use of hydrophilic polymers to efficiently coat conventional nanoparticles surface opened the possibility of creates new types of nanoparticles. ${ }^{3}$ In this way, coating conventional nanoparticles with surfactants, in order to obtain a long-circulating carrier, has been the first strategy used to direct tumor targeting in vivo. In addition, the synthesis of biocompatible super paramagnetic materials has long been of interest in biomedical applications including magnetic resonance imaging for clinical diagnosis, magnetic drug targeting, hyperthermia as anticancer strategy, and enzyme immobilization. ${ }^{4}$

Among the characteristics of magnetic nanoparticles: easily penetration through the cell or bacterial membrane, exhibition of super paramagnetic phenomena, hysteresis loss in the alternative magnetic field for hyperthermia, and availability for chemical binding; the last one confer a high grade of versatility for different purposes such as cell separation, enzyme and protein immobilization, immunoassays, RNA/DNA purification, etc. ${ }^{5-8}$ Some authors have even proposed the preparation of multifunctional nanoparticles or nanoplatforms with three or more functions (multi-gene vector, drug carrier, and heating properties for hyperthermia). ${ }^{9,10}$ On the other hand, antisense oligodeoxynucleotide (ODN) technique is an important area of research in cancer-associated treatment. ODN are linear strands of nucleotide bases synthesized in a sequence complementary to the protein-encoding mRNAs and acts inhibiting the synthesis of specific proteins. This type of therapy has less toxic side-effects and allows the selective targeting of cancer cells. ${ }^{11}$

To enhance the efficiency of antisense therapy, ODN must be developed containing multiple binding sites. ${ }^{12}$ Shen et al. ${ }^{13}$ reported the use of a 19-mer antisense ODN, complementary to a template region of the telomerase RNA, coupled with iron magnetic nanoparticles. The use of this combination resulted in the depression of telomerase activity and led to cell apoptosis. The association of ODN with nanoparticles prevents the ODN degradation by nucleases increasing the cellular uptake and prolonged the half-life inside cells and thus with the advantage of increasing the efficiency of antisense activity. ${ }^{1}$ Recently, antisense ODN has been labeled with iron nanoparticles for tumor antisense gene detection with magnetic resonance gene imaging. This novel molecular probe could be potentially useful as magnetic resonance targeting contrast enhancing agent to diagnose tumors at an early stage ${ }^{14}$ see in (Figure 1).

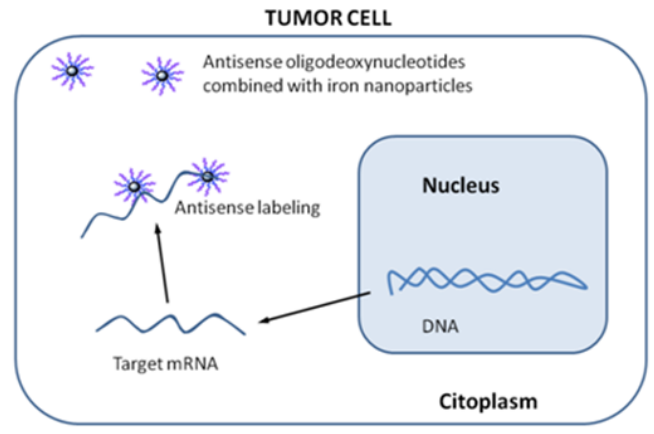

Figure I Gene detection by MRI, traduction blockage by antisense labelling and hyperthermic treatment by magnetic excitation of nanoparticles.

\section{Conclusion}

The applications of nanoparticles in biomedicine (nanobiotechnology) are increasing over time, being nowadays a 
promising area in research and health care, with future innovative methods for diagnostic and treatment of cancer.

\section{Acknowledgements}

None.

\section{Conflict of interest}

The author declares no conflict of interest.

\section{References}

1. Brigger I, Dubernet C, Couvreur P. Nanoparticles in cancer therapy and diagnosis. Adv Drug Deliv Rev. 2002;54(5):631-651.

2. Wang JM, Xiao BL, Zheng JW, et al. Effect of targeted magnetic nanoparticles containing 5-FU on expression of bcl-2, bax and caspase 3 in nude mice with transplanted human liver cancer. World J Gastroenterol. 2007; 13(23):3171-3175.

3. Storm G, Belliot SO, Daemen T, et al. Surface modification of nanoparticles to oppose uptake by the mononuclear phagocyte system. Adv Drug Deliv Rev. 1995;17:31-48.

4. Cheng FY, Su CH, Yang YS, et al. Characterization of aqueous dispersions of $\mathrm{Fe}_{3} \mathrm{O}_{4}$ nanoparticles and their biomedical applications. Biomaterials. 2005;26(7):729-738.

5. Hütten A, Sudfeld D, Ennen I, et al. New magnetic nanoparticles for biotechnology. J Biotechnol. 2004;112(1-2):47-63.

6. Glöckl G, Brinkmeier V, Aurich K, et al. Development of a liquid phase immunoassay by time-dependent measurements of the transient magnetooptical birefringence using functionalized magnetic nanoparticles. $J$ Magn Magn Mater. 2005;289:480-483.
7. Bai S, Guo Z, Liu W, et al. Resolution of $( \pm)$-menthol by immobilized Candida rugosa lipase on superparamagnetic nanoparticles. Food Chem. 2006;96(1):1-7.

8. Chiang CL, Sung CS. Purification of transfection-grade plasmid DNA from bacterial cells with superparamagnetic nanoparticles. J Magn Magn Mater. 2006;302(1):7-13.

9. Xiao X, He Q, Huang K. Possible magnetic multifunctional nanoplatforms in medicine. Med Hypotheses. 2007;68(3):680-682.

10. Ciofani G, Riggio C, Raffa V, et al. A bi-modal approach against cancer: Magnetic alginate nanoparticles for combined chemotherapy and hyperthermia. Med Hypotheses. 2009;73(1):80-82.

11. Stahel RA, Zangemeister-Wittke V. Antisense oligonucleotides for cancer therapy an overview. Lung Cancer. 2003;41(1s):581-588.

12. Rubenstein M, Tsui P, Guinan P. Antisense oligonucleotides as specific chemotherapeutic delivery agents: A new type of bifunctional antisense. Med Hypotheses Res. 2009;5:57-61.

13. Shen $\mathrm{HB}$, Long $\mathrm{DH}$, Zhu $\mathrm{LZ}$, et al. Magnetic force microscopy analysis of apoptosis of HL-60 cells induced by complex of antisense oligonucleotides and magnetic nanoparticles. Biophys Chem. 2006;122(1):1-4.

14. Wen MW, Li B, Ouyang Y, et al. Preparation and quality test of superparamagnetic iron oxide labeled antisense oligodeoxynucleotide probe: A preliminary study. Ann Biomed Eng. 2009;37(6):1240-1250. 